\title{
OLED display technology
}

\author{
Askari Mohammad Bagher \\ Department of Physics, Payame Noor University, PO Box 19395-3697 Tehran, Iran
}

Email address:

MB_Askari@yahoo.com

\section{To cite this article:}

Askari Mohammad Bagher. OLED Display Technology. American Journal of Optics and Photonics. Vol. 2, No. 3, 2014 , pp. $32-36$. doi: 10.11648/j.ajop.20140203.13

\begin{abstract}
OLEDs are solid-state devices composed of thin films of organic molecules that create light with the application of electricity. OLEDs can provide brighter, crisper displays on electronic devices and use less power than conventional light-emitting diodes (LEDs) or liquid crystal displays (LCDs) used today. OLEDs are made by placing thin films of organic (carbon based) materials between two conductors. When electrical current is applied, a bright light is emitted. The OLED materials emit light and do not require a backlight (unlike LCDs). Each pixel is a small light-emitting diode, in fact. OLEDs emit light they do not require a backlight and so are thinner and more efficient than LCD displays (which do require a white backlight).In this paper we investigate the characteristics of OLED.
\end{abstract}

Keywords: Organic Light-Emitting Diode, OLED

\section{Introduction}

An organic light-emitting diode (OLED) consists of several semiconducting organic layers sandwiched between two electrodes, at least one of them being transparent. Two simplified device structures are shown in figure 1: a one side emitting device (left) and a transparent one which emits light both upwards and downwards (right). The device is fabricated by sequentially depositing organic layers on a conducting substrate followed by another conducting electrode. A common device structure comprises a glass substrate coated with indium tin oxide (ITO) as transparent anode and a thin, opaque metal fi $\mathrm{lm}$ as cathode. The organic stack including the electrodes is usually thinner than $1 \mu \mathrm{m}$. Two classes of organic materials are commonly used for organic light-emitting devices: polymeric substances and so-called "small molecule materials" which do not exhibit any orientating property and therefore form amorphous films. One interesting aspect of organics-based optoelectronics is the possibility to use simple screen printing or wet deposition techniques for cost-effective fabrication of large-area devices. Nowadays, this applies only to polymeric organics, whereas evaporating techniques still have to be applied for small molecules.

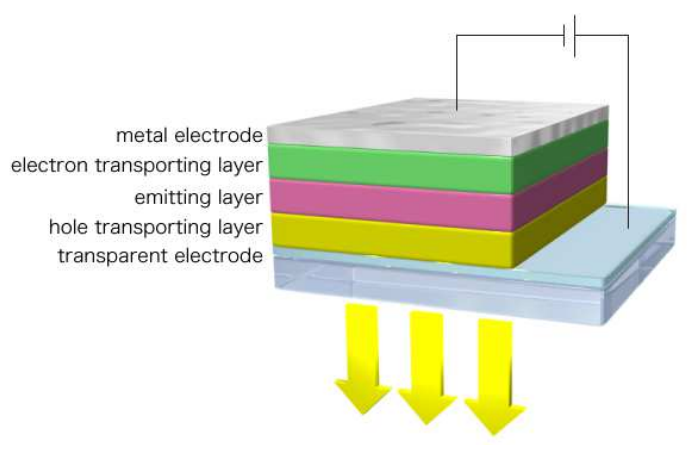

Figure 1. Simplify structures of an organic light-emitting device

\section{Characteristics of OLED}

Below, we outlined number of characters on OLED. We intend to review each of these characteristics. The following can be cited, including OLED characters.

\subsection{Flat Panels Thin and Flexible}

OLED is an emerging display technology that enables beautiful and efficient displays and lighting panels. Thin OLEDs are already being used in many mobile devices and TVs, and the next generation of these panels will be flexible and bendable. When we talk about flexible OLEDs, it's important to understand what that means exactly. A 
flexible OLED is based on a flexible substrate which can be plastic, metal or flexible glass. The plastic and metal panels will be light, thin and very durable - in fact they will be virtually shatter-proof. It is estimated that the first range of devices to use a flexible display won't be flexible at all. While the manufacturer may bend the display or curve it around a non-flat surface, the final user will not be able to actually bend the device. Still it will have several advantages: these displays will be lighter, thinner and much more durable compared to glass based displays. Second generation flexible OLED devices may indeed be flexible to the final user. Finally, when the technology is ready, we may see OLED panels that you can fold, bend or stretch. This may create all sorts of exciting designs that will enable large displays to be placed in a mobile device and only be opened when required. Fabricating a flexible OLED display is very challenging. The two major challenges are the backplane and the encapsulation [1].

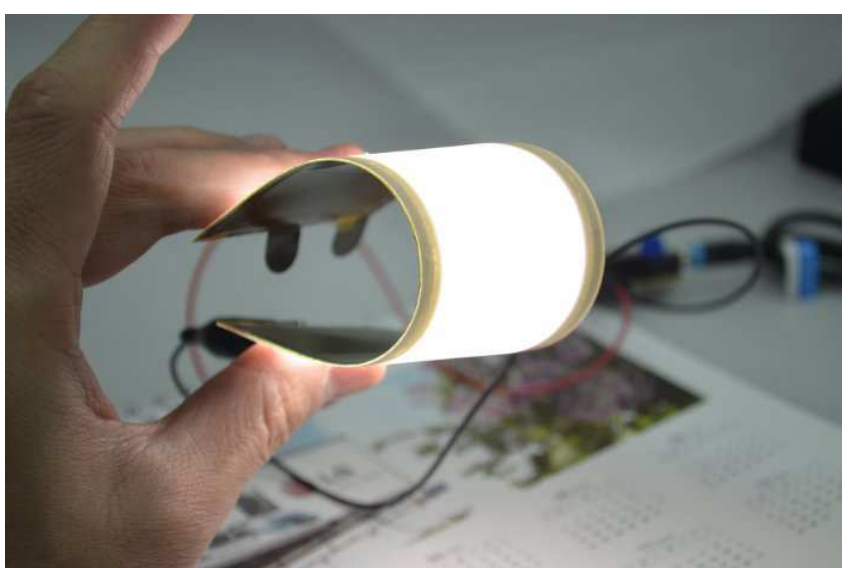

Figure 2. (www.oled-info.com)

1: current encapsulation technology is Vita's multi-layer technology which is very slow (the panel has to enter the evaporation chamber 6 times). Some companies are working on single-layer barriers and better encapsulation processes, but these haven't been scaled to mass production yet.

2: According to reports, companies is still using LTPS for their plastic based panels, and the production process for LTPS is too hot for the plastic substrate (it will melt) and currently deposits the LTPS transistors on glass and the glass is later delaminated. Some companies display is using an Oxide-TFT which should be easier to produce on a flexible substrate.

OLEDs can also be used to make white lighting panels. OLED is a diffuse area lighting source with unique characteristics. While OLED lighting is still in its infancy, many believe that flexible OLED lighting panels may provide designers with a new lighting source that will create stunning designs.

\subsection{Light Weight}

Light has always been an indispensable part of our everyday lives. The ultimate reference for artificial light is of course, natural light. Among all high-efficiency/eco-friendly light, OLEDs have the closest spectral distributions to natural light, even in its most natural state. One of the main reasons that a company Chem. is dedicated to producing OLED is to spread its good quality light into the world. OLEDs possess novel and unique characteristics while being the world's most human-friendly light source whose quality of light will eventually represent a higher quality of life. An OLED emits light due to the electroluminescence of thin films of organic semiconductors approximately $100 \mathrm{~nm}$ thick. Regular OLEDs are usually fabricated on a glass substrate, but by replacing glass with a flexible plastic such as polyethylene terephthalate (PET) [2] among others, [3] OLEDs can be made both bendable and lightweight. OLED lighting is attracting attention as the next generation lighting having such unprecedented features as thin, light weight, and surface light source. In addition, it features high energy efficacy, no scalding surface, and reduced environmental footprint since it is mercury-free unlike fluorescent lightings. Furthermore, OLED lighting technology is mild to eyes and friendly to people, since it is free of ultra-violet rays.

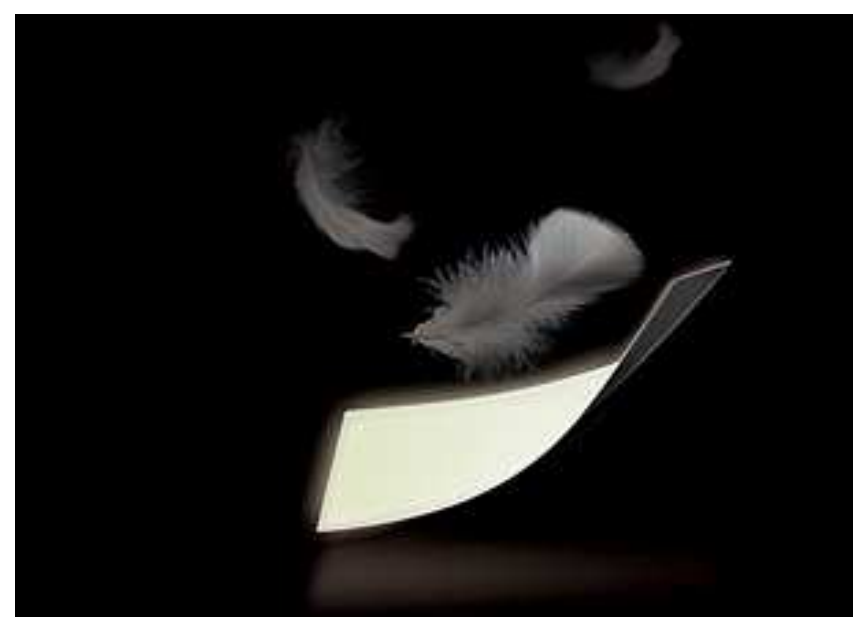

Figure 3. (www.konicaminolta.com)

\subsection{Low Cost}

Researchers worldwide continue search for better luminescent materials for OLED manufacturing. Two new compounds with europium complexes display in their class record high luminescence efficiencies in red, and their properties enable faster, low cost manufacturing of thin OLED films. The processing technology is also different. Currently, amorphous oxide semiconductor TFTs are prepared by a process that requires a large vacuum chamber and a costly coating procedure. UCLA researchers used a solution process, akin to applying a coat of paint to an object and then baking it in an oven. "The improved mobility represents a breakthrough for solution-processed metal oxide TFTs, device's performance is comparable to that of commercial TFTs, but it can be produced at a much lower cost." 


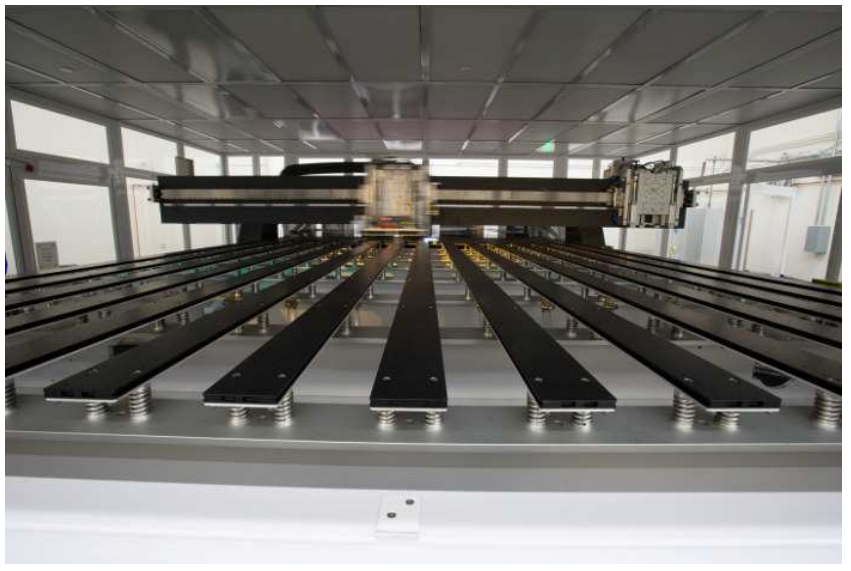

Figure 4. (www.oled-info.com)

\subsection{Backlighting and Bright}

OLEDs are brighter than LEDs. Because the organic layers of an OLED are much thinner than the corresponding inorganic crystal layers of an LED, the conductive and emissive layers of an OLED can be multilayered. Also, LEDs and LCDs require glass for support, and glass absorbs some light. OLEDs do not require glass [4].

LED stands for light-emitting diode. These are little solid-state devices that make light because of the movement of electrons through a semi-conductor. LEDs are relatively small compared to compact fluorescent and incandescent light bulbs, but they can get extremely bright. However, LEDs aren't small enough to be used as the pixels of a television - they're way too big for that. That's why LEDs are only used as the backlight for LCD televisions. OLEDs do not require backlighting like LCDs. LCDs work by selectively blocking areas of the backlight to make the images that you sees, while OLEDs generate light themselves. Because OLEDs do not require backlighting, they consume much less power than LCDs (most of the LCD power goes to the backlighting). This is especially important for battery-operated devices such as cell phones.

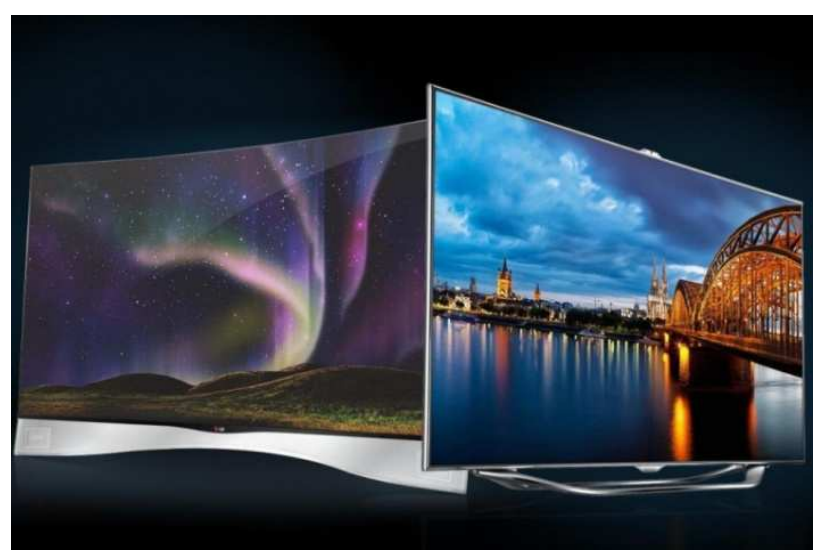

Figure 5. (http://www.digitaltrends.com/)

\subsection{Long Life}

As the graph below indicates, recent improvements in the OLED allow some of our OLEDs to run more efficiently with higher outputs. When run through a lower current, a longer life for the OLED will result. This improvement in lifetime allows for the technology to be used in more applications and provides further protection of product longevity. Standard off-the-shelf displays are available as well as custom solutions for high volume application This improvement in lifetime allows for the technology to be used in more applications and provides further protection of product longevity. Standard off-theshelf displays are available as well as custom solutions for high volume applications [5].
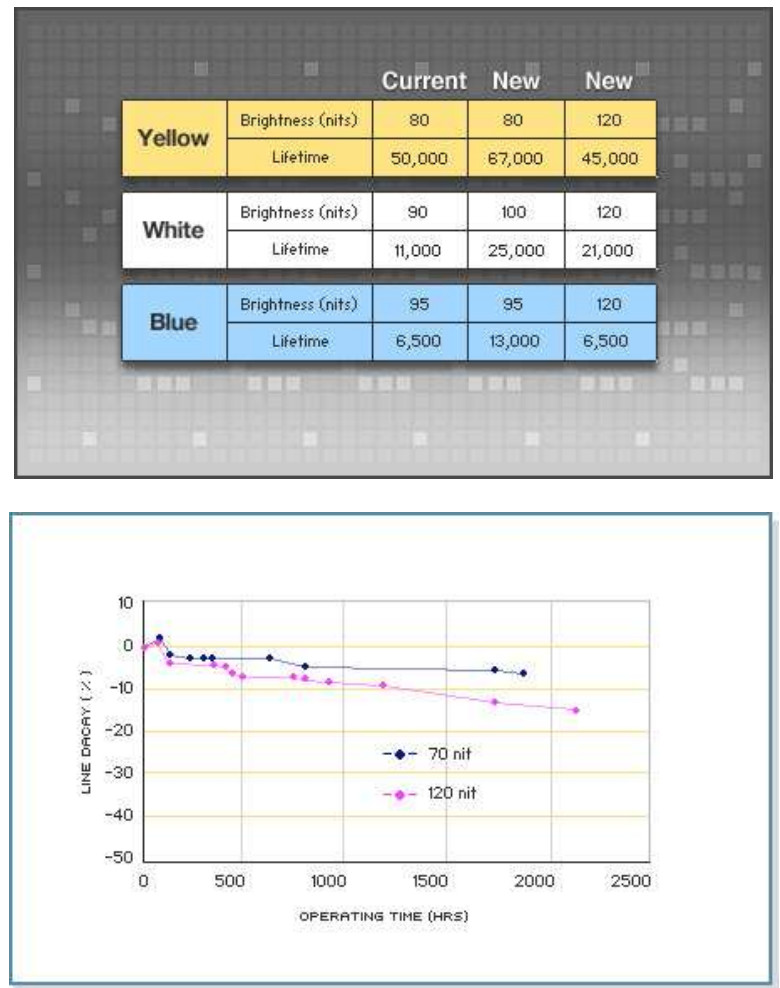

Figure 6. (http://usmicroproducts.com)

\subsection{Low Voltage}

Efficient and low-voltage top-emitting OLED using surface-modified $\mathrm{Ag}$ anode is reported. By inducing a thin silver oxide at the surface of $\mathrm{Ag}$, whole injection from $\mathrm{Ag}$ anodes into OLEDs is largely enhanced yet with rather high reflectivity retained. Top-emitting devices using such surface-modified Ag anode show device characteristics competitive with those of a bottom-emitting device using the indium tin oxide anode. OLED materials are designed to generate light with high efficiency at a low voltage in a stable device structure. Typically $70 \%$ of the light is trapped in the OLED. Efficient extraction of generated light is the next key step to improve OLED efficiency. Nova led introduced a new product series using dedicated out coupling Materials to increase light extraction [6]. 


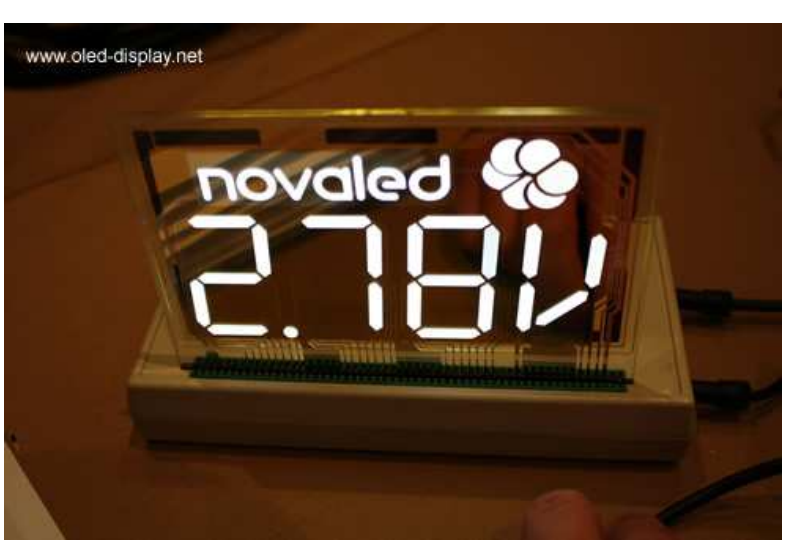

Figure 7. (www.oled-display.net)

\subsection{Good Luminous Efficiency}

OLEDs are capable of emitting any color including white when a voltage is applied by combining organic materials that emit various colors such as red, green, and blue. The soft surface luminescence makes OLEDs suitable for lighting a wide area, and along with the benefits of being not overly bright when viewed directly and a thin and lightweight structure, they are receiving attention as the next generation light source. Lighting devices equipped with the high efficiency OLEDs are considered to achieve a luminous efficiency comparable to or higher than that of fluorescent lamps (approx. 60-80 lm/W) or LED lighting fixtures (approx. $80 \mathrm{~lm} / \mathrm{W}$ ) currently available in the market. Further, a wide variety of applications are expected for OLED devices, including use in general lighting. White OLED lighting will create new value in people's daily lives and has great potential for realizing a comfortable and ecological lifestyle.

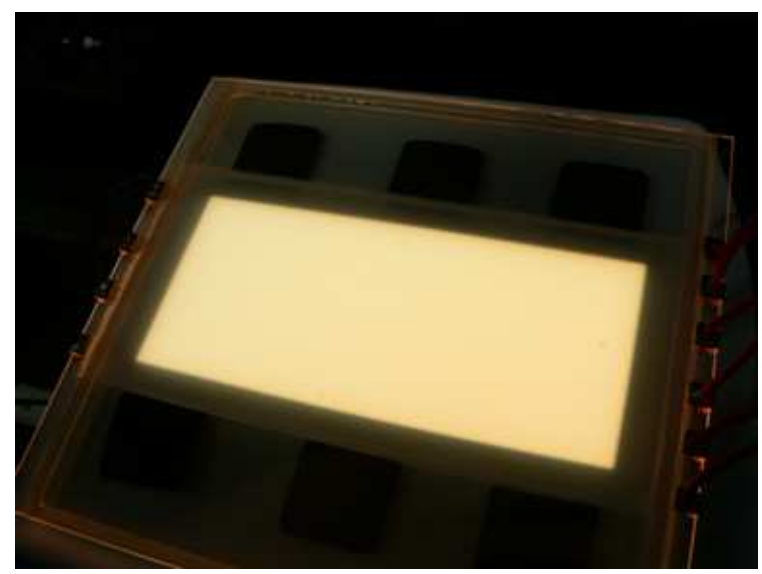

Figure 8. (http://panasonic.co.jp) (The Newly Developed White OLED with the World's Highest Luminous Efficiency)

According to the sum company the $15 \times 15 \mathrm{~cm}$ OLED panel was able to achieve better luminous efficacy with the use of new in-house developed blue phosphor. In addition, the company developed light extractive technology enabling light to be extracted from organic layers to increase quantum efficiency.

\section{1- $\quad$ Large color range}

OLEDs can enable a greater artificial contrast ratio (both dynamic range and static, measured in purely dark conditions) and a wider viewing angle compared to LCDs because OLED pixels emit light directly. OLED pixel colors appear correct and un shifted, even as the viewing angle approaches $90^{\circ}$ from normal. Additionally, as the OLED material used to produce blue light degrades significantly more rapidly than the materials that produce other colors; blue light output will decrease relative to the other colors of light. This variation in the differential color output will change the color balance of the display and is much more noticeable than a decrease in overall luminance.[7] This can be avoided partially by adjusting color balance, but this may require advanced control circuits and interaction with the user, which is unacceptable for some users. More commonly, though, manufacturers optimize the size of the $R, G$ and $B$ sub pixels to reduce the current density through the sub pixel in order to equalize lifetime at full luminance. For example, a blue sub pixel may be $100 \%$ larger than the green sub pixel. The red sub pixel may be $10 \%$ smaller than the green. Improvements to the efficiency and lifetime of blue OLEDs are vital to the success of OLEDs as replacements for LCD technology. Considerable research has been invested in developing blue OLEDs with high external quantum efficiency as well as a deeper blue color.[8] [9] External quantum efficiency values of $20 \%$ and $19 \%$ have been reported for red $(625 \mathrm{~nm})$ and green $(530 \mathrm{~nm})$ diodes, respectively.[10] [11] However, blue diodes $(430 \mathrm{~nm})$ have only been able to achieve maximum external quantum efficiencies in the range of $4 \%$ to $6 \%$.[12]

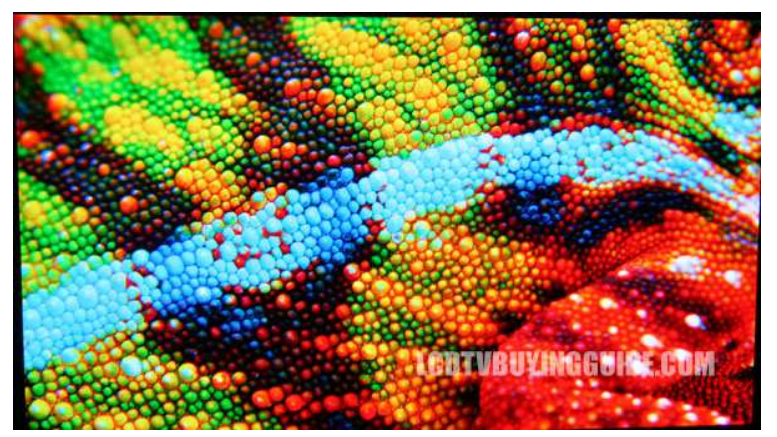

Figure 9. (lcdtvbuyingguide.com)

\section{Reference}

[1] http://www.oled-info.com/flexible-oled

[2] Gustafsson, G.; Cao, Y.; Treacy, G. M.; Klavetter, F.; Colaneri, N.; Heeger, A. J. (1992). "Flexible light-emitting diodes made from soluble conducting polymers". Nature 357 (6378): 477. Bibcode:1992Natur.357..477G. Doi:10.1038/357477a0.

[3] MacDonald, W. A. (2004). "Engineered films for display technologies". Journal of Materials Chemistry 14: 4-10. Doi:10.1039/B310846P. 
[4] http://electronics.howstuffworks.com

[5] http://usmicroproducts.com

[6] http://www.novaled.com

[7] "Ageless OLED". Retrieved 2009-11-16.

[8] Shen, Jiun Yi; Lee, Chung Ying; Huang, Tai-Hsiang; Lin, Jiann T.; Tao, Yu-Tai; Chien, Chin-Hsiung; Tsai, Chiitang (2005). "High Tg blue emitting materials for electroluminescent devices". Journal of Materials Chemistry (free text) 15 (25): 2455. doi:10.1039/b501819f.

[9] Kim, Seul Ong; Lee, Kum Hee; Kim, Gu Young; Seo, Ji Hoon; Kim, Young Kwan; Yoon, Seung Soo (2010). "A highly efficient deep blue fluorescent OLED based on diphenylaminofluorenylstyrene-containing emitting materials". Synthetic Metals 160 (11-12): 1259.
[10] Jabbour, G. E.; Kawabe, Y.; Shaheen, S. E.; Wang, J. F.; Morrell, M. M.; Kippelen, B.; Peyghambarian, N. (1997). "Highly efficient and bright organic electroluminescent devices with an aluminum cathode". Applied Physics Letters 71 (13): 1762.

[11] Mikami, Akiyoshi; Koshiyama, Tatsuya; Tsubokawa, Tetsuro (2005). "High-Efficiency Color and White Organic Light-Emitting Devices Prepared on Flexible Plastic Substrates". Japanese Journal of Applied Physics 44: 608.

[12] Mikami, Akiyoshi; Nishita, Yusuke; Iida, Yoichi (2006). "35-3: High Efficiency Phosphorescent Organic LightEmitting Devices Coupled with Lateral Color-Conversion Layer". SID Symposium Digest of Technical Papers 37: 1376. 